\title{
A!
}

This is an electronic reprint of the original article.

This reprint may differ from the original in pagination and typographic detail.

Aarva, Anja; Laurila, Tomi; Caro, Miguel A.

Doping as a means to probe the potential dependence of dopamine adsorption on carbonbased surfaces

Published in:

Journal of Chemical Physics

DOI:

$10.1063 / 1.4986521$

Published: 21/06/2017

Document Version

Publisher's PDF, also known as Version of record

Please cite the original version:

Aarva, A., Laurila, T., \& Caro, M. A. (2017). Doping as a means to probe the potential dependence of dopamine adsorption on carbon-based surfaces: A first-principles study. Journal of Chemical Physics, 146(23), [234704]. https://doi.org/10.1063/1.4986521

This material is protected by copyright and other intellectual property rights, and duplication or sale of all or part of any of the repository collections is not permitted, except that material may be duplicated by you for your research use or educational purposes in electronic or print form. You must obtain permission for any other use. Electronic or print copies may not be offered, whether for sale or otherwise to anyone who is not an authorised user. 


\section{Doping as a means to probe the potential dependence of dopamine adsorption on carbon-based surfaces: A first-principles study}

Anja Aarva, Tomi Laurila, and Miguel A. Caro

Citation: The Journal of Chemical Physics 146, 234704 (2017); doi: 10.1063/1.4986521

View online: https://doi.org/10.1063/1.4986521

View Table of Contents: http://aip.scitation.org/toc/jcp/146/23

Published by the American Institute of Physics

\section{Articles you may be interested in}

Accurate schemes for calculation of thermodynamic properties of liquid mixtures from molecular dynamics simulations

The Journal of Chemical Physics 145, 244504 (2016); 10.1063/1.4973001

Assessment of two hybrid van der Waals density functionals for covalent and non-covalent binding of molecules

The Journal of Chemical Physics 146, 234106 (2017); 10.1063/1.4986522

First principles study of 2D layered organohalide tin perovskites

The Journal of Chemical Physics 146, 234703 (2017); 10.1063/1.4985054

Evaluating continuum solvation models for the electrode-electrolyte interface: Challenges and strategies for improvement

The Journal of Chemical Physics 146, 084111 (2017); 10.1063/1.4976971

Simplified continuum solvent model with a smooth cavity based on volumetric data

The Journal of Chemical Physics 141, 174108 (2014); 10.1063/1.4900838

Revised self-consistent continuum solvation in electronic-structure calculations

The Journal of Chemical Physics 136, 064102 (2012); 10.1063/1.3676407

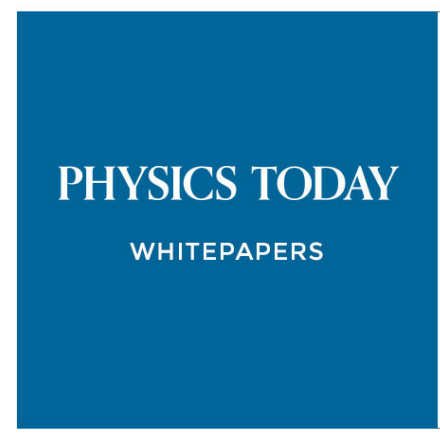

ADVANCED LIGHT CURE ADHESIVES

Take a closer look at what these environmentally friendly adhesive systems can do
READ NOW

PRESENTED BY

Q. MASTERBOND' 


\title{
Doping as a means to probe the potential dependence of dopamine adsorption on carbon-based surfaces: A first-principles study
}

\author{
Anja Aarva, ${ }^{1, a)}$ Tomi Laurila, ${ }^{1}$ and Miguel A. Caro ${ }^{1,2}$ \\ ${ }^{1}$ Department of Electrical Engineering and Automation, School of Electrical Engineering, Aalto University, \\ Espoo, Finland \\ ${ }^{2}$ COMP Centre of Excellence in Computational Nanoscience, Department of Applied Physics, Aalto University, \\ Espoo, Finland
}

(Received 22 February 2017; accepted 5 June 2017; published online 21 June 2017)

\begin{abstract}
In this work, we study the adsorption characteristics of dopamine (DA), ascorbic acid (AA), and dopaminequinone $\left(\mathrm{DA}_{\mathrm{ox}}\right)$ on carbonaceous electrodes. Our goal is to obtain a better understanding of the adsorption behavior of these analytes in order to promote the development of new carbon-based electrode materials for sensitive and selective detection of dopamine in vivo. Here we employ density functional theory-based simulations to reach a level of detail that cannot be achieved experimentally. To get a broader understanding of carbonaceous surfaces with different morphological characteristics, we compare three materials: graphene, diamond, and amorphous carbon (a-C). Effects of solvation on adsorption characteristics are taken into account via a continuum solvent model. Potential changes that take place during electrochemical measurements, such as cyclic voltammetry, can also alter the adsorption behavior. In this study, we have utilized doping as an indirect method to simulate these changes by shifting the work function of the electrode material. We demonstrate that $s p^{2}$ and $s p^{3}$-rich materials, as well as a-C, respond markedly different to doping. Also the adsorption behavior of the molecules studied here differs depending on the surface material and the change in the surface potential. In all cases, adsorption is spontaneous, but covalent bonding is not detected in vacuum. The aqueous medium has a large effect on the adsorption behavior of $\mathrm{DA}_{\mathrm{ox}}$, which reaches its highest adsorption energy on diamond when the potential is shifted to more negative values. In all cases, inclusion of the solvent enhances the charge transfer between the slab and $\mathrm{DA}_{\mathrm{ox}}$. Largest differences in adsorption energy between DA and AA are obtained on graphene. Gaining better understanding of the behavior of the different forms of carbon when used as electrode materials provides a means to rationalize the observed complex phenomena taking place at the electrodes during electrochemical oxidation/reduction of these biomolecules. Published by AIP Publishing. [http://dx.doi.org/10.1063/1.4986521]
\end{abstract}

\section{INTRODUCTION}

Dopamine (DA), which belongs to a group of catecholamines, is an important neurotransmitter. The determination of DA levels in vivo would help in understanding several neurological disorders such as Parkinson's disease, schizophrenia, and depression. As a molecule, DA is electrochemically active; thus electrochemical techniques can be utilized to detect dopamine levels in vitro and even in vivo. ${ }^{1,2}$ Carbon-based materials, in particular, amorphous carbon (aC), have been shown to be promising candidates as electrode materials for neural sensing. ${ }^{3-5}$ They are biocompatible and resistant to bacterial adhesion. Unfortunately, they cannot distinguish between ascorbic acid (AA) and DA. Ascorbic acid is one of the main interfering elements when DA measurements are carried out in vivo on carbon-based electrodes. ${ }^{4,5}$ It oxidizes approximately at the same potential range, and in vivo it is present in much lager concentrations than DA. ${ }^{4,6}$ Both DA and AA are inner-sphere redox systems. This means that their oxidation/reduction behavior strongly

$\overline{{ }^{a)} \text { Electronic mail: anja.aarva@gmail.com }}$ depends on the electrode surface chemistry and the adsorption characteristics of the molecules. ${ }^{7}$ It has indeed been suggested that adsorption plays an important role in the dopamine oxidation reaction path ${ }^{7,8}$ although the nature of the expected adsorption on different carbon surfaces is far from clear.

The applied potential and the different forms that the different molecules adopt during the oxidation reaction path can alter the adsorption behavior and interactions with the electrode. Suggestions for the reaction path of DA oxidation on carbonaceous electrodes are given in Refs. 3, 5, and 9 and for AA oxidation in Ref. 9. Both of these electrochemical reactions can be followed by chemical reactions. ${ }^{2,3,5,9}$ It has been proposed that the oxidized form of dopamine can further react chemically, and polymerize and agglomerate on the surface of the electrode, which can be detected as degradation of the electrode performance. ${ }^{2,3,5}$ Thus, optimal adsorption characteristics would allow the analyte to adsorb strongly enough to facilitate electrochemical oxidation, but not too strongly, to prevent degradation caused by the products of the chemical reactions that follow oxidation. It could also be possible to exploit the possible differences in the adsorption 
behavior of AA and DA on various carbon surfaces to enhance the differentiation between the two molecules.

Unlike other forms of carbon, amorphous carbon has a very complex structure, and it contains carbon that can be bonded in various ways $\left(s p^{1}, s p^{2}\right.$, and $\left.s p^{3}\right)$ and can form several different kinds of ring structures. ${ }^{10,11}$ The material also typically contains elements other than carbon, such as hydrogen and oxygen. These elements can be found as functional groups present on the surface already after processing. ${ }^{12}$ Although these carbon-based materials have already been characterized in detail $1^{4,6,10-13}$ and their electrochemical performance has been widely studied, $3,4,6,12$ there still remains a lack of fundamental understanding of the basic phenomena taking place at the atomic level. Understanding in more depth the adsorption behavior of AA and DA on carbon-based electrodes would enable us to develop new surface modification schemes to induce selectivity and increase sensitivity towards these molecules.

In this work, we utilize density functional theory (DFT) based computational methods to study DA, dopaminequinone $\left(\mathrm{DA}_{\mathrm{ox}}\right)$, and $\mathrm{AA}$ adsorption on different carbonaceous surfaces. In order to understand the chemistry between DA and carbonaceous surfaces even better, $\mathrm{DA}_{\mathrm{ox}}$, which is the oxidation product of $\mathrm{DA},{ }^{2,5}$ has been included in this study owing to its expected importance in the passivation of the electrodes when electrochemical oxidation of DA is utilized for DA detection. In the case of these three molecules, favorable molecular adsorption is considered to be the key to induce charge transfer that takes place in electrochemical oxidation/reduction. Although the electron transport has been shown to have a role in certain electrochemical reactions taking place on carbonaceous electrodes, its role becomes comparable to the electron transfer only after the film thickness becomes relatively large. ${ }^{14}$ Thus, a detailed study of effects of electron transport on the adsorption of DA, $\mathrm{AA}$, and $\mathrm{DA}_{\mathrm{ox}}$ on carbon based electrodes is left beyond the scope of this work. In this study, we focus on inspecting the situation before and after adsorption. To draw a more realistic picture of the adsorption process, however, we incorporate the effect of electrode potential changes. These changes take place during experimental measurements when cyclic voltammetry is used. These measurements are most commonly carried out in aqueous solution, which we have incorporated to the simulations by using an effective dielectric medium. ${ }^{15-17}$ Given the intrinsic difficulties to include the effect of surface potential in DFT simulations, we approach the problem indirectly via shift of the Fermi level through the introduction of dopants. Replacing a variable number of carbon atoms by either boron or nitrogen, we induce changes in the work function of the surfaces. In practice, this situation is equivalent to tuning the surface potential externally. The aim of this work is not to study the effect of doping atoms on adsorption, and therefore, dopant atom substitution needs to be carried out far away from the adsorption site so as to not influence the process. In other words, doping is only used as an indirect method to assess the effect of electrode potential variability on the adsorption characteristics of $\mathrm{DA}, \mathrm{DA}_{\mathrm{ox}}$, and AA.

\section{SIMULATION DETAILS}

\section{A. Model systems}

Our model systems are constructed to represent three different carbon-based electrode surfaces: graphene, diamond, and a-C. We work within the supercell approach, whereby the different "infinite" surfaces are represented by finite-size slabs with periodic boundary conditions imposed on the plane of the surface and fixed boundary conditions along the direction perpendicular to it. ${ }^{18}$ For the graphene sheets, two supercell sizes were used to check the effect of system size on the convergence of its properties. The two configurations used correspond to $6 \times 6 \times 1$ and $9 \times 9 \times 1$ primitive unit cells for the "small" (72 carbon atoms) and "large" (162 atoms) graphene slabs, respectively. Note that, among other improvements, increasing the in-plane dimensions of the slabs helps reduce the spurious electrostatic interaction between the different periodic replicas of the molecules once they have been added to the system. The size of the a-C supercell used is approximately $12 \times 12 \AA$ (in-plane) and the slab is $6 \AA$ thick (perpendicular to the surface), for a total of 125 carbon atoms. The reconstructed (D341) diamond slab consists of 144 carbon atoms. We employ $3 \times 4$ primitive unit cells of the $2 \times 1$ reconstruction of the diamond (111) surface ${ }^{19}$ and 6 atomic monolayers of thickness. Dangling bonds at the bottom are passivated with hydrogens. All the supercells were set up so that there was an adequate amount of vacuum above the system to ensure that the energy is converged to the correct value. In vacuum calculations, dipole corrections ${ }^{20}$ were applied in the direction perpendicular to the surface to ensure the correct asymptotic behavior of the electrostatic potential away from the surface. The carbon structures employed in this work are depicted in Fig. 1.

The a-C surface was generated as explained in detail in Ref. 10. In essence, bulk a-C samples were generated using

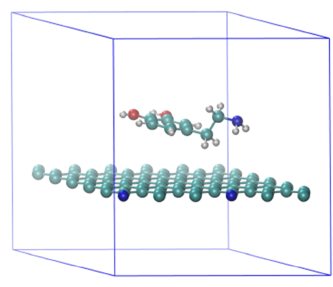

(a)

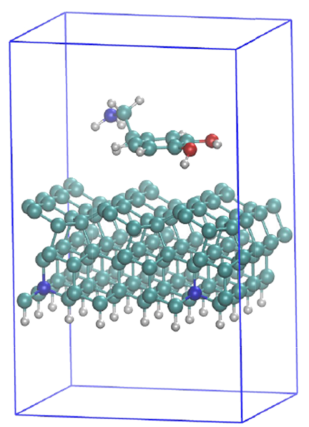

(b)

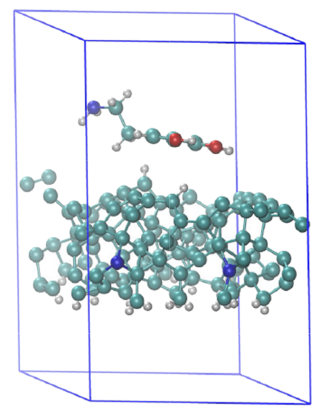

(c)
FIG. 1. Examples of (a) a graphene, (b) a diamond, and (c) an amorphous carbon system: these systems are doped with two nitrogens placed at the side or in the bottom of the slab. The analyte, DA, is placed on top of the surface. 
random initialization followed by geometry optimization. After this, a pressure correction was performed through box rescaling followed again by further geometry optimization. This method allows us to generate a-C networks with $s p^{3}$ content versus density relations in closer agreement with the experiment than previous approaches. ${ }^{10,21}$ After this bulk optimization, the periodic boundary constraint along the vertical axis is lifted. The bottom of the supercell is "anchored" by letting it bind to a diamond substrate, which allows maintaining the bulk-like properties in the interior of the slab. The top of the slab is allowed to reconstruct via geometry optimization, leading to a dramatic increase in $s p^{2}$ hybridized carbon atoms right at the surface. The resulting slab is too large to carry out the numerous calculations to study adsorption characteristics required for this work. Therefore, the bottom part of the slab is removed by cleaving, and the resulting dangling bonds are passivated with hydrogen. The approach has been shown to yield a good convergence of the work function and residual forces even for relatively small slab sizes. ${ }^{10,22}$ The a-C slab used in this paper is the $2.82 \mathrm{~g} / \mathrm{cm}^{3}$ slab of Ref. 10 cleaved at a depth of $6 \AA$ below its uppermost $\mathrm{C}$ atom. This particular slab contains two highly reactive sites on its surface. These dangling bonds are part of the a-C structure. ${ }^{10}$ In practice, it can be anticipated that these very active $s p^{1}$ sites would be passivated by hydrogen or other functional groups before the electrode surface is brought in contact with the analyte, either during the fabrication process or soon afterwards. The activity of these particular sites was established by inspecting the local density of states (LDOS), that is, the DOS projected onto the atomic orbitals of the carbon atoms at those particular sites. It was critical to identify these sites since they can strongly bond with the different molecules when brought in contact with them, giving large and unrealistic adsorption or binding energies. In this work, we passivate these two sites with hydrogen although other possibilities could involve, for instance, oxygen and carboxyl groups. Here we have chosen hydrogen because of its small size and because it does not cause steric hindrance between the molecule and the carbon surface. Functionalized surfaces are beyond the scope of this work. Here we are interested in studying the interactions between the analytes and pristine carbon surfaces.

To shift the position of the Fermi level and therefore simulate the effect of varying electrode potential, we introduce dopants. We choose between boron and nitrogen, which are the most commonly employed dopants in carbon networks. Doping is realized by replacing one carbon atom by a dopant atom, as far away as possible from the chosen adsorption site. Since boron has one valence electron less than carbon, it works as an electron acceptor and removes the electron density from the carbon network, lowering the Fermi level. Analogously, nitrogen works as an electron donor, raising the Fermi level. In the case of a-C, the dopant atoms are placed at the bottom of the slab [Fig. 1(c)] at $s p^{3}$ sites in order to force them to behave similarly to how they would in a diamond lattice. The structure is constrained so that the geometry of the bottom is fixed. Thus, dopant atoms and their neighbors are not allowed to move. In reconstructed diamond, the dopant atoms are also at the bottom of the slab [Fig. 1(b)]. In the case of graphene, the dopants are placed in sites with in-plane positions as far away as possible from the location of the molecule [Fig. 1(a)]. To prevent strongly perturbing the electronic structure of the carbon network (beyond the intended shift of the Fermi level), in all cases, dopant atoms are positioned away from each other, such that two dopants never share a nearest neighbor.

The electronic density of states (DOS) profiles for borondoped, undoped, and nitrogen-doped a-C, diamond, and graphene are shown in Fig. 2, and the effect of doping on slab work function at different dopant-to-carbon ratios (in the absence of adsorbed molecules) is shown in Fig. 3. From Fig. 2, we observe that the main effect of doping is indeed the intended shift in the Fermi level position. From Fig. 3, where negative doping values correspond to nitrogen and positive values to boron, we observe that relatively large potential windows, up to $\sim 1.00 \mathrm{eV}$ and up to $\sim 1.42 \mathrm{eV}$, can be obtained for diamond and graphene, respectively. However, in the case of amorphous carbon, the window is much narrower. This stems from the much higher DOS present in the a-C pseudogap (Fig. 2) compared to diamond or graphene. Beyond certain dopant to carbon ratios, approximately $\pm 2.0 \%$ for both
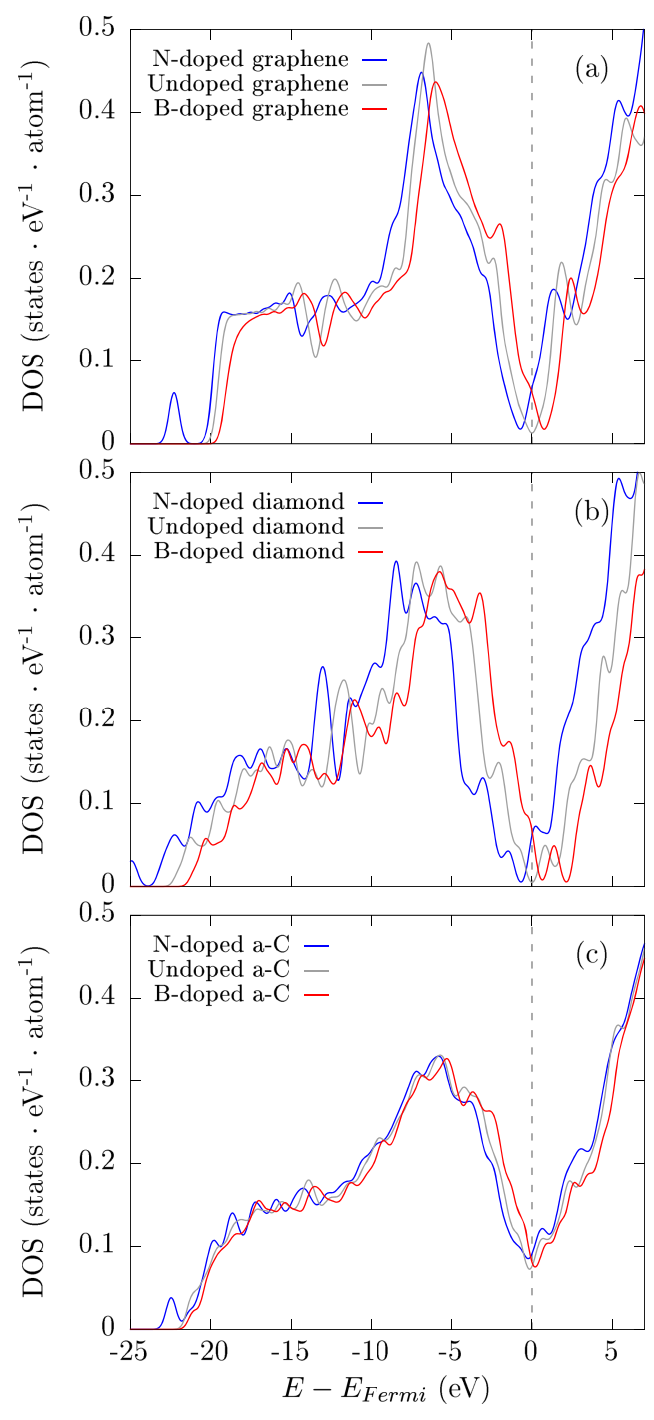

FIG. 2. The electronic density of states (DOS) for boron-doped, undoped, and nitrogen-doped graphene (a), diamond (b), and a-C (c). The original position of the Fermi level is indicated by the dashed line. 


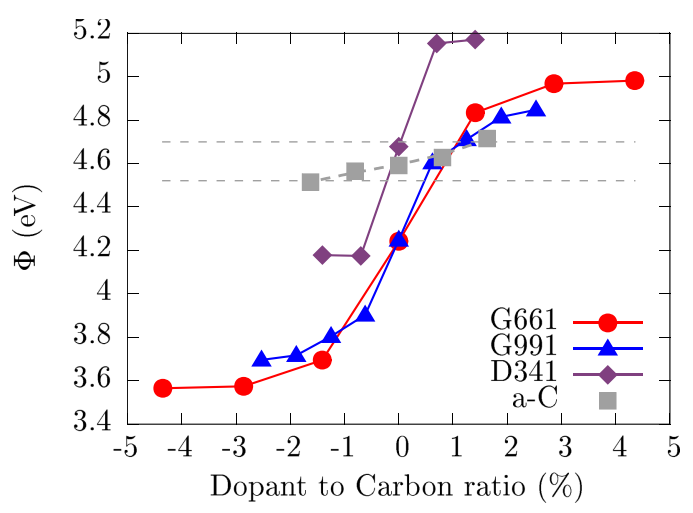

FIG. 3. Work function as a function of dopant to carbon ratio for a-C, diamond (D341), and two graphene systems of different sizes. The graphene supercells used are $6 \times 6 \times 1$ and $9 \times 9 \times 1$ primitive unit cells (G661 and G991, respectively). Nitrogen and boron concentrations are represented on the horizontal axis with negative and positive values, respectively.

$6 \times 6 \times 1$ and $9 \times 9 \times 1$ graphene, and approximately $\pm 1.4 \%$ and $\pm 1.6 \%$ for diamond and a-C, respectively, further addition of dopants does not significantly increase/decrease the work function any further because of the growing DOS around the gap/pseudogap of the material.

Finally, the analyte molecule (DA, $\mathrm{DA}_{\mathrm{ox}}$ or AA) is placed on the surface, and the system is let to relax until it has found the minimum energy configuration with respect to geometry and electronic structure. The structures for DA and AA were collected from PubChem, ${ }^{23}$ and $\mathrm{DA}_{\mathrm{ox}}$ was created from DA by removing two electrons and two protons, i.e., in total two hydrogens, in accordance with the known reaction scheme. ${ }^{5}$ All molecules are let to relax without any symmetry constraints. The analyte molecule is initially set approximately $3 \AA$ above the slab, so that it has room to move up and down, and it is not forced within the primary bonding distance of the surface.

\section{B. Computational methods}

Density functional theory (DFT) ${ }^{24}$ enables to study the system from first principles, i.e., the interactions between the analyte and the electrode surface can be studied without any previous knowledge of the outcome. This feature is particularly useful especially in the present case, where the process leading to DA oxidation at the electrode surfaces is not known experimentally. Here we explain the technical details of our simulations.

The GPAW suite ${ }^{25}$ was used to perform self-consistent Kohn-Sham (KS) density functional theory ${ }^{24,26}$ calculations. The exchange-correlation density functional used in the calculations is the Perdew-Burke-Ernzerhof (PBE) generalizedgradient approximation (GGA). ${ }^{27}$ The grid spacing in real space used for the representation of wave functions, electron density, potential, etc., is $0.16 \AA$. $k$-space integration was performed using different Monkhorst-Pack (MP) grids $^{28}$ for diamond, a-C, and graphene supercells: $1 \times 2 \times 1 k$-point sampling for diamond, $2 \times 2 \times 1 k$-point sampling for a-C and the $9 \times 9 \times 1$ graphene slab, and $3 \times 3 \times 1 k$-point sampling for the $6 \times 6 \times 1$ graphene slab. van der Waals corrections were taken into account via the method developed by Tkatchenko and
Scheffler. ${ }^{29}$ All of the amorphous carbon calculations were carried out with spin polarization because of the existence of local (atomic) magnetic moments in a-C. ${ }^{21}$ Smearing of the occupation numbers $(0.1 \mathrm{eV})$, based on Fermi-Dirac distributions, was used in order to help convergence. ${ }^{25}$

In addition to studying the interactions between the molecules and the surfaces in vacuum at different potential values, solvation effects were taken into consideration via a continuum solvent model ${ }^{15,16}$ implemented in GPAW by Held and Walter. ${ }^{17}$ In this model, the molecule and the surface are encapsulated in a smooth cavity, and to simulate aqueous solution, the remaining volume of the cell is "filled" with a dielectric medium whose dielectric constant corresponds to that of bulk water, as water is the most common solvent used in the electrochemical measurements. This method does not only allow us to compare adsorption energies calculated in vacuum to those calculated in solution but also enables us to study differences in charge transfer characteristics in vacuum and in solution.

To get an idea of charge-transfer characteristics between slabs and molecules, in vacuum as well as in the dielectric medium, we used the Bader partitioning scheme developed by Henkelman et al. ${ }^{30}$ This tool allows assigning electronic charges to individual atoms in the systems that comprise the surface and the adsorbed molecule. Net charges of the adsorbed molecules were obtained by adding up the charges of the individual atoms in that particular molecule.

Adsorption energies were obtained as the difference between the energy of the whole relaxed system (slab plus adsorbed molecule) $E_{\text {tot }}$ and the sum of the energies of the surface $\left(E_{\text {surface }}\right)$ and the molecule alone (for DA, $\left.E_{\mathrm{DA}}\right)$ in vacuum/in the dielectric medium, i.e., total energies were calculated both in vacuum and in solution. For instance, the adsorption energy for $\mathrm{DA}, E_{\mathrm{ad}}^{\mathrm{DA}}$, is

$$
E_{\mathrm{ad}}^{\mathrm{DA}}=E_{\mathrm{tot}}-\left(E_{\text {surface }}+E_{\mathrm{DA}}\right)
$$

where

$$
E_{\mathrm{tot}}=E_{\text {surface }+\mathrm{DA}} \text {. }
$$

When calculating the energy of the molecule, the same box used in the supercell calculations was used. In this way, the error due to electrostatic (e.g., dipole-dipole) self-interaction of the molecules under periodic boundary conditions can be minimized through error cancellation (approximately the same spurious interaction is present when the molecules are adsorbed on the surface).

To calculate the work function, the energy difference between the Fermi level and the vacuum level is computed. Since a-C slabs are asymmetric and may have an intrinsic out-of-plane dipole moment, the vacuum level used for work function calculation is always computed on the side of the slab where the molecule adsorbs. In addition, the size of the molecular dipole is comparable to the overall supercell dipole due to finite-size restrictions. If there is charge transfer between the molecule and the substrate upon adsorption, it can also affect the region where the Fermi level of the whole supercell lies. Consequently, the presence of the molecule may significantly affect the work function of the system, through both the dipoledriven shift in the vacuum level and the charge transfer-driven shift of the Fermi level. Dipole and charge redistribution can 


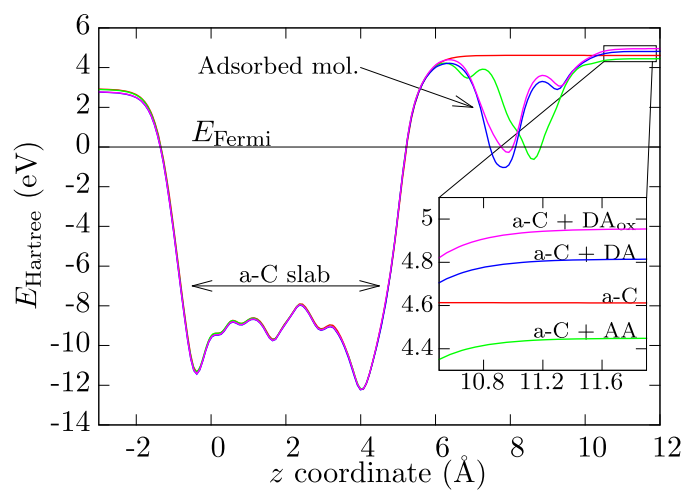

FIG. 4. The electrostatic potentials of a-C systems with different adsorbed molecules.

in turn affect each other such that the work function of the slab is affected by the presence of the adsorbed molecule in a non-trivial way. In Fig. 4, we show how the presence of the different adsorbed molecules has a large impact on the work function of the overall system, up to $+300 \mathrm{meV}$ in the case of $\mathrm{DA}_{\mathrm{ox}}$ adsorption (see the inset of Fig. 4). These changes are of same order of magnitude as the overall potential windows that can be realized within the present doping scheme (Fig. 3), and need to be accounted for. To correct for this, we report work function differences between the calculations at zero and finite doping ratios, where the adsorbed molecule is present in both cases. This will be discussed further in Sec. III.

\section{RESULTS AND DISCUSSION}

As we showed in Fig. 3, the work functions of graphene and diamond show a strong correlation with dopant concentration. Amorphous carbon shows the same behavior, but the window is much narrower. Also, the work functions of the undoped surfaces differ: $\Phi_{\mathrm{a}-\mathrm{C}}=4.60 \mathrm{eV}, \Phi_{\text {diamond }}=4.68 \mathrm{eV}$, and $\Phi_{\text {graphene }}=4.25 \mathrm{eV}$. The graphene results compare well with the experimental and computational characterization of boron- and nitrogen-doped graphene by Panchakarla et al. ${ }^{31}$ However, as it was described previously, we are not interested in the effect of doping on adsorption as such, but doping is used as an indirect method to shift the Fermi level (and hence the work function) of the surfaces studied here.

As previously discussed, adding a molecule on the surface within the supercell approach can significantly shift the work function of the system as a whole. Here, we use the change in work function, $\Delta \Phi$, for the adsorbed system at doping concentration $x$ with respect to the reference case of an adsorbed molecule at zero doping concentration,

$$
\Delta \Phi(x)=\Phi(x)-\Phi(0) .
$$

The adsorption energies, $E_{\mathrm{ad}}$, of dopamine, dopaminequinone, and ascorbic acid on graphene (both $6 \times 6 \times 1$ and $9 \times 9 \times 1$ slabs) as a function of $\Delta \Phi$, in vacuum, are presented in Fig. 5(a). The purpose of this work is not to present definitive values for $E_{\text {ad }}$ but to obtain the correct qualitative behavior in order to estimate trends in the interaction between the analytes and the surfaces. In all cases, adsorption energies are negative for the molecules studied here; thus their adsorption occurs spontaneously. DA and $\mathrm{DA}_{\mathrm{ox}}$ adsorb more strongly
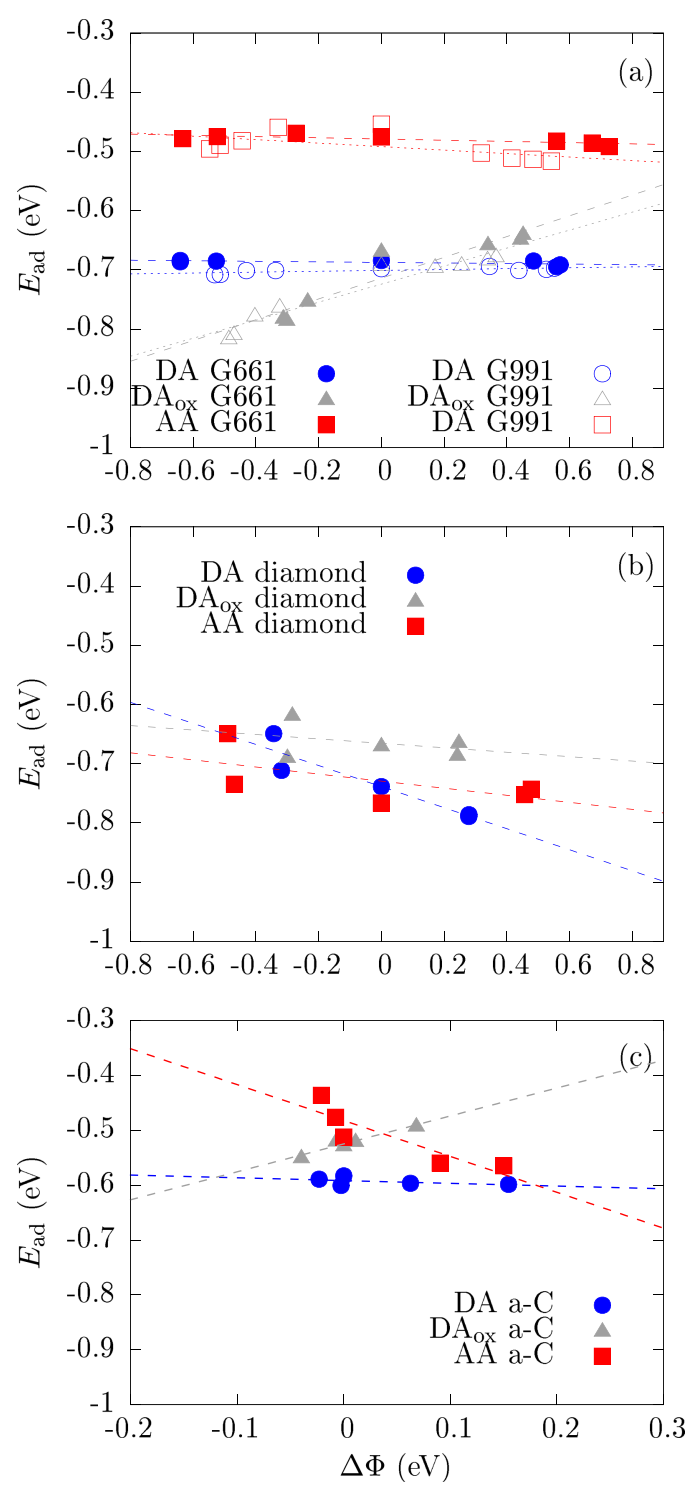

FIG. 5. Adsorption energies, $E_{\mathrm{ad}}$, of $\mathrm{DA}, \mathrm{DA}_{\mathrm{Ox}}$, and $\mathrm{AA}$ in vacuum as a function of the change in work function, $\Delta \Phi$, on graphene (G661 and G991), on diamond, and on a-C are depicted in (a)-(c), respectively.

than AA. Interestingly, for DA and AA adsorbed on graphene, the adsorption energy has no appreciable dependence on the change in work function, $\Delta \Phi$. For $\mathrm{DA}_{\mathrm{ox}}$, on the other hand, the trend of adsorption energy versus work function is very clear. By decreasing the work function or, equivalently, decreasing the surface potential, the adsorption energy quickly moves towards more negative values. In other words, $\mathrm{DA}_{\mathrm{ox}}$ adsorption on graphene is clearly favored by negative potentials.

The adsorption energies of $\mathrm{DA}, \mathrm{DA}_{\mathrm{Ox}}$, and $\mathrm{AA}$ on diamond are presented in Fig. 5(b). In this case, the adsorption energies of the molecules studied here are all negative and very similar. DA and AA show a slightly descending trend in adsorption energies towards increased surface potential.

The adsorption energies of DA, $\mathrm{DA}_{\mathrm{ox}}$, and $\mathrm{AA}$ on $\mathrm{a}-\mathrm{C}$ in vacuum are depicted in Fig. 5(c). The adsorption energies of the molecules are all negative also in this case. $E_{\text {ad }}^{\mathrm{AA}}$ becomes slightly more negative as the surface potential increases, whereas $E_{\text {ad }}^{\mathrm{DA}_{\mathrm{ox}}}$ shows a descending trend towards negative potentials. 

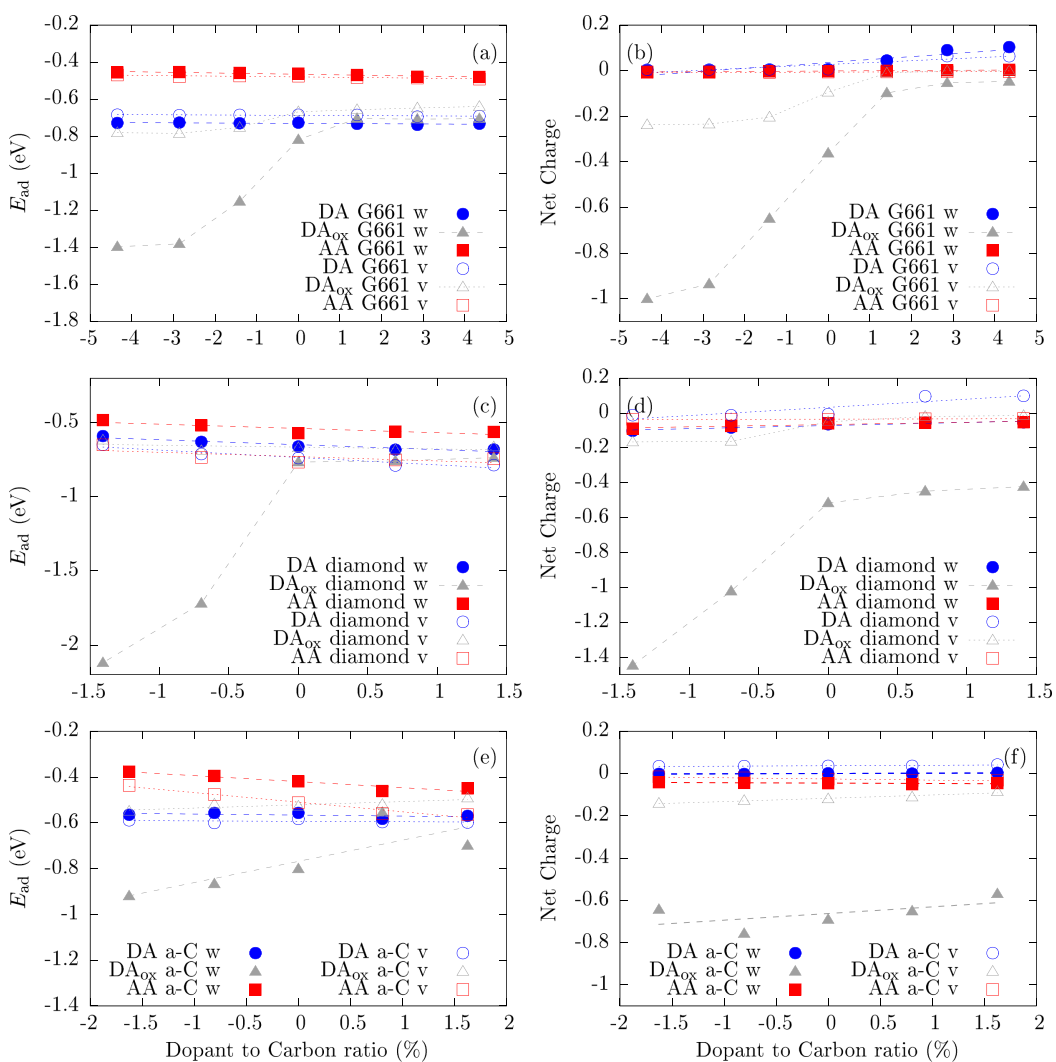

FIG. 6. Adsorption energies, $E_{\mathrm{ad}}$, of DA, DA $\mathrm{ox}_{\mathrm{ox}}$, and AA as a function of dopant to carbon ratio (nitrogen and boron concentrations are represented on the horizontal axis with negative and positive values, respectively) on graphene (G661), on diamond, and on a-C, in vacuum (v) and in dielectric medium (w), are depicted in (a), (c), and (e), respectively. Net charges of the molecules, obtained with Bader analysis, on graphene, on diamond, and on a-C as a function of dopant to carbon ratio, in vacuum (v) and in dielectric medium (w), are depicted in (b), (d), and (f), respectively.
In Fig. 6, we present a comparison of adsorption energies, $E_{\mathrm{ad}}$, of $\mathrm{DA}, \mathrm{DA}_{\mathrm{ox}}$, and $\mathrm{AA}$ in vacuum and in solution as a function of dopant to carbon ratio. Here we have chosen to use the dopant to carbon ratio instead of the work function since, within this framework, the work function cannot be straightforwardly computed in solution. Nitrogen concentrations are depicted as negative values and boron concentrations as positive values on the horizontal axis. Net charges of the molecules, obtained with Bader analysis, in vacuum (v) and in dielectric medium (w), are also depicted as a function of dopant to carbon ratio.

Since the Fermi level is varied by adding dopant atoms and not by removing or adding electrons, the system remains overall neutral. Also, all isolated molecules and all isolated surfaces are neutral, but when the molecule is brought to the surface and the electronic structure is relaxed, the electron density is redistributed between the surface and the molecule. The net charges of the molecules on diamond [Fig. 6(d)] and on a-C [Fig. 6(f)] are in the same order of magnitude as on graphene [Fig. 6(b)] and show a behavior similar to that on graphene with the exception that $\mathrm{DA}_{\mathrm{ox}}$ accepts the electron density from the slab also when the surface potential is shifted towards more positive values, and the net charge of the molecule remains negative within the whole potential window. It can be observed in all three cases that as nitrogen concentration in the slab increases, and thus $\Delta \Phi$ is shifted to more negative values, $E_{\mathrm{ad}}^{\mathrm{DA}_{\mathrm{ox}}}$ becomes more negative. The net charge of $\mathrm{DA}_{\mathrm{ox}}$ becomes more negative as well, i.e., electron density moves from the slab towards $\mathrm{DA}_{\mathrm{ox}}$. This effect becomes even more clear in the dielectric medium where at the same time $E_{\text {ad }}^{\mathrm{DA}_{\text {ox }}}$ becomes notably more negative. $E_{\mathrm{ad}}^{\mathrm{DA}}$ and $E_{\mathrm{ad}}^{\mathrm{AA}}$ do not change as dramatically in the presence of solvent effects, compared to vacuum. In fact, the effect of changing the work function of the slab on the charge transfer in the case of $\mathrm{DA}_{\mathrm{ox}}$ is so large that it can be clearly detected when the charge density before and after adsorption is visualized (Fig. 7).

When the adsorption energies and the charge transfer between the molecules and the slabs are examined by inspecting the net charges of the adsorbed molecules (Fig. 6), it can be seen that the adsorption behavior of DA and AA seems to be fairly independent of the changes in the potential, whereas the adsorption of $\mathrm{DA}_{\mathrm{ox}}$ becomes stronger when the potential is shifted towards more negative values. Solvation energies of DA and $\mathrm{DA}_{\mathrm{ox}},-0.54 \mathrm{eV}$ and $-0.64 \mathrm{eV}$, calculated with the continuum solvation method are relatively similar. The corresponding value for AA is $-0.96 \mathrm{eV}$. As expected based on the large electronegativity of oxygen, the exposed oxygen atoms in $\mathrm{DA}_{\mathrm{ox}}$ withdraw charge from the surface. The inclusion of the aqueous medium further enhances this effect. Especially on graphene, $E_{\mathrm{ad}}^{\mathrm{DA}}$, in vacuum as well as in solution, correlates strongly with the net charge of the molecule. On diamond, we detect the same trend in charge transfer, but in vacuum $E_{a d}^{\mathrm{DA}_{\mathrm{ox}}}$ seems to be independent of $\Delta \Phi$. Yet, when the system is solvated, $E_{\mathrm{ad}}^{\mathrm{DA}}$ ox reacts strongly to shifts in the Fermi level and the correlation with net charge is clear. Similar trends can be seen on a-C within the potential window that was achieved with a-C. In practice, the charge transfer between the adsorbing molecule and a-C surface is likely to be affected by functional groups. This strong interaction between carbonaceous surfaces and $\mathrm{DA}_{\mathrm{ox}}$ supports the idea that the electrode performance can be deteriorated by agglomeration of the reaction products of the electrochemical oxidation and the following 


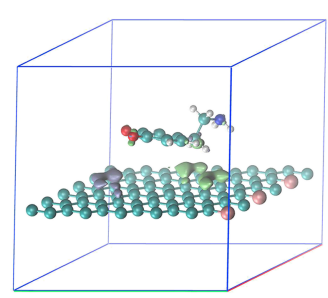

(a)

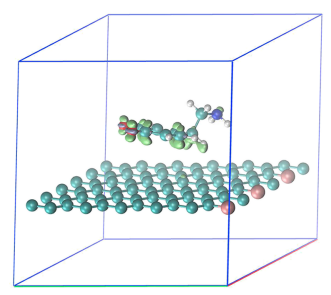

(d)

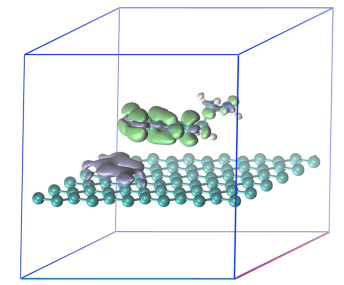

(b)

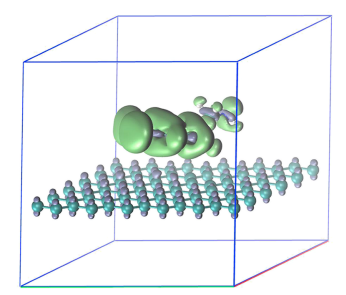

(e)

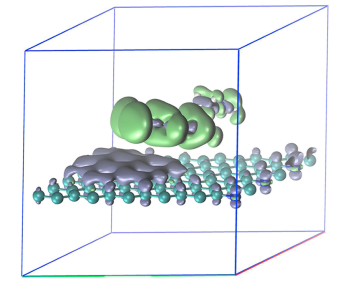

(c)

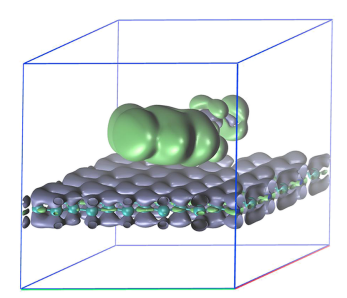

(f)
FIG. 7. Dopaminequinone on boron-doped, undoped, and nitrogen-doped graphene in vacuum (a)-(c) and in dielectric medium (d)-(f). Upon adsorption, charge density flows from the violet to the green region. Dopaminequinone on graphene has been chosen here as an example system for visualization because it is perhaps the simplest system discussed in this study and thus provides the clearest image of the phenomena. chemical reactions. At the same time, since it has been stated that the oxidation/reduction of these molecules is accompanied by favorable adsorption, ${ }^{7}$ it would be reasonable to suggest that $E_{\mathrm{ad}}^{\mathrm{DA}}$ becomes more negative as the molecule is being reduced.

\section{CONCLUSIONS}

In this work, we have introduced doping as a method to simulate changes in the surface potential via changing the Fermi level (and hence the work function) of carbonaceous electrodes. All of the materials discussed here, graphene, diamond, and amorphous carbon, respond to doping analogously by the shift in work function (Fig. 3). With graphene and diamond, relatively large potential windows can be achieved, whereas in the case of a-C, the window remains narrow due to the much higher DOS present in the pseudogap region.

We have also studied the adsorption of three molecules, dopamine, dopaminequinone, and ascorbic acid, on these carbonaceous surfaces and examined the effect of changing the surface potential on the adsorption behavior in vacuum as well as in aqueous medium. Adsorption energies are negative in all cases, and in vacuum, they remain within the same magnitude as hydrogen bonding. Thus, in vacuum, covalent bonding is not observed. Incorporating the effects of solvent as an effective dielectric medium has very little impact on the adsorption characteristics of DA and AA. The adsorption energies of these two molecules are very similar, which can partly explain why it is difficult to distinguish between DA and AA in electrochemical measurements. $E_{\text {ad }}^{\mathrm{DA}}$ and $E_{\mathrm{ad}}^{\mathrm{AA}}$ differ substantially only on graphene. The difference between $E_{\mathrm{ad}}^{\mathrm{DA}}$ and $E_{\mathrm{ad}}^{\mathrm{AA}}$ acquired on graphene suggests that $s p^{2}$-rich surfaces could improve the selectivity of the electrode.

In the case of $\mathrm{DA}_{\mathrm{Ox}}$, adsorption energies change dramatically with the addition of the solvent. In vacuum on diamond, $E_{\mathrm{ad}}^{\mathrm{DA}}$ is more negative than $E_{\mathrm{ad}}^{\mathrm{DA}}$, and in addition, $E_{\mathrm{ad}}^{\mathrm{DA}}$ does not become stronger when the potential is shifted to more negative values. Thus, the adsorption behavior of $\mathrm{DA}_{\mathrm{ox}}$ in vacuum indicates that $s p^{3}$-rich materials could be more resistant towards the electrode deactivation caused by agglomeration of oxidation products than $s p^{2}$-rich materials. In contrast, in dielectric medium, $\mathrm{DA}_{\mathrm{ox}}$ is more strongly adsorbed on diamond than on graphene, and the charge transfer between the slab and the molecule is enhanced.

In reality, the electrode surface is seldom purely $s p^{2}$ or $s p^{3}$-like, and carbon can form very complex amorphous structures having several different carbon sites ${ }^{10,11}$ and their reactivity varies. Thus, calculations carried out on graphene or on diamond only cannot be directly generalized for all carbonaceous surfaces. By studying and comparing all of these three materials, a deeper understanding of the surface chemistry of carbonaceous electrodes can be achieved. Furthermore, understanding the differences between $s p^{2}$ - or $s p^{3}$-rich materials could facilitate the development of electrode surfaces towards sensitive and selective sensing of biomolecules such as dopamine.

\section{ACKNOWLEDGMENTS}

Funding from Academy of Finland (Project No. 285526) is gratefully acknowledged. The computational resources provided for this project by Aalto University's Science-IT through the Triton cluster as well as CSC-IT Center for Science trough the Taito cluster are also gratefully acknowledged.

${ }^{1}$ R. M. Wightman, L. J. May, and A. C. Michael, "Detection of dopamine dynamics in the brain," Anal. Chem. 60, 769A-793A (1988).

${ }^{2}$ S. Chumillas, M. C. Figueiredo, V. Climent, and J. M. Feliu, "Study of dopamine reactivity on platinum single crystal electrode surfaces," Electrochim. Acta 109, 577-586 (2013).

${ }^{3}$ T. Palomäki, S. Chumillas, S. Sainio, V. Protopopova, M. Kauppila, J. Koskinen, V. Climent, J. M. Feliu, and T. Laurila, "Electrochemical reactions of catechol, methylcatechol and dopamine at tetrahedral amorphous carbon (ta-C) thin film electrodes," Diamond Relat. Mater. 59, 30-39 (2015).

${ }^{4}$ S. Sainio, T. Palomäki, S. Rhode, M. Kauppila, O. Pitkänen, T. Selkälä, G. Toth, M. Moram, K. Kordas, J. Koskinen, and T. Laurila, "Carbon nanotube (CNT) forest grown on diamond-like carbon (DLC) thin films significantly improves electrochemical sensitivity and selectivity towards dopamine," Sens. Actuators, B 211, 177-186 (2015).

${ }^{5}$ A. N. Patel, S. Tan, T. S. Miller, J. V. Macpherson, and P. R. Unwin, "Comparison and reappraisal of carbon electrodes for the voltammetric detection of dopamine," Anal. Chem. 85, 11755-11764 (2013).

${ }^{6}$ S. Sainio, T. Palomäki, N. Tujunen, V. Protopopova, J. Koehne, K. Kordas, J. Koskinen, M. Meyyappan, and T. Laurila, "Integrated carbon nanostructures for detection of neurotransmitters," Mol. Neurobiol. 52, 859-866 (2015). 
${ }^{7}$ S. H. DuVall and R. L. McCreery, "Control of catechol and hydroquinone electron-transfer kinetics on native and modified glassy carbon electrodes," Anal. Chem. 71, 4594-4602 (1999).

${ }^{8}$ S. H. DuVall and R. L. McCreery, "Self-catalysis by catechols and quinones during heterogeneous electron transfer at carbon electrodes," J. Am. Chem. Soc. 122, 6759-6764 (2000).

${ }^{9}$ M. R. Deakin, P. M. Kovach, K. J. Stutts, and R. M. Wightman, "Heterogeneous mechanisms of the oxidation of catechols and ascorbic acid at carbon electrodes," Anal. Chem. 58, 1474-1480 (1986).

${ }^{10}$ M. A. Caro, R. Zoubkoff, O. Lopez-Acevedo, and T. Laurila, “Atomic and electronic structure of tetrahedral amorphous carbon surfaces from density functional theory: Properties and simulation strategies," Carbon 77, 1168 (2014).

${ }^{11}$ J. Robertson, "Diamond-like amorphous carbon," Mater. Sci. Eng. R 37, 129-281 (2002).

${ }^{12}$ S. Sainio, D. Nordlund, M. A. Caro, R. Gandhiraman, J. Koehne, N. Wester, J. Koskinen, M. Meyyappan, and T. Laurila, "Correlation between $\mathrm{sp}^{3}$-to-sp ${ }^{2}$ ratio and surface oxygen functionalities in tetrahedral amorphous carbon (ta-C) thin film electrodes and implications of their electrochemical properties," J. Phys. Chem. C 120, 8298-8304 (2016).

${ }^{13}$ T. Laurila, S. Sainio, H. Jiang, T. Palomäki, O. Pitkänen, K. Kordas, and J. Koskinen, "Multi-walled carbon nanotubes (MWCNTs) grown directly on tetrahedral amorphous carbon (ta-C): An interfacial study," Diamond Relat. Mater. 56, 54-59 (2015).

${ }^{14}$ T. Palomäki, N. Wester, M. A. Caro, S. Sainio, V. Protopopova, J. Koskinen, and T. Laurila, "Electron transport determines the electrochemical properties of tetrahedral amorphous carbon (ta-c) thin films," Electrochim. Acta 225, 1-10 (2017).

${ }^{15}$ V. M. Sánchez, M. Sued, and D. A. Scherlis, "First-principles molecular dynamics simulations at solid-liquid interfaces with a continuum solvent," J. Chem. Phys. 131, 174108 (2009).

${ }^{16}$ W. Im, D. Beglov, and B. Roux, "Continuum solvation model: Computation of electrostatic forces from numerical solutions to the Poisson-Boltzmann equation," Comput. Phys. Commun. 111, 59-75 (1998).

${ }^{17}$ A. Held and M. Walter, "Simplified continuum solvent model with a smooth cavity based on volumetric data,” J. Chem. Phys. 141, 174108 (2014).
${ }^{18}$ G. Makov and M. C. Payne, "Periodic boundary conditions in ab initio calculations," Phys. Rev. B 51, 4014 (1995).

${ }^{19}$ S. Iarlori, G. Galli, F. Gygi, M. Parrinello, and E. Tosatti, "Reconstruction of the diamond (111) surface," Phys. Rev. Lett. 69, 2947 (1992).

${ }^{20}$ J. Neugebauer and M. Scheffler, "Adsorbate-substrate and adsorbateadsorbate interactions of $\mathrm{Na}$ and K adlayers on Al (111)," Phys. Rev. B 46, 16067 (1992).

${ }^{21}$ T. Laurila, S. Sainio, and M. A. Caro, "Hybrid carbon based nanomaterials for electrochemical detection of biomolecules," Prog. Mater. Sci. 88, 499_ 594 (2017).

${ }^{22}$ M. A. Caro, R. Zoubkoff, O. Lopez-Acevedo, and T. Laurila, "Corrigendum to "Atomic and electronic structure of tetrahedral amorphous carbon surfaces from density functional theory: Properties and simulation strategies' [Carbon 77 (2014) 1168-1182]," Carbon 82, 612-613 (2015).

${ }^{23}$ See https://pubchem.ncbi.nlm.nih.gov/ for information about PubChem database.

${ }^{24}$ R. M. Martin, Electronic Structure: Basic Theory and Practical Methods (Cambridge University Press, 2004).

${ }^{25}$ See https://wiki.fysik.dtu.dk/gpaw/ for installation and documentation.

${ }^{26}$ W. Kohn and L. J. Sham, "Self-consistent equations including exchange and correlation effects," Phys. Rev. 140, A1133 (1965).

${ }^{27}$ J. P. Perdew, K. Burke, and M. Ernzerhof, "Generalized gradient approximation made simple," Phys. Rev. Lett. 77, 3865 (1996).

${ }^{28}$ H. J. Monkhorst and J. D. Pack, "Special points for Brillouin-zone integrations," Phys. Rev. B 13, 5188 (1976).

${ }^{29}$ A. Tkatchenko and M. Scheffler, "Accurate molecular Van der Waals interactions from ground-state electron density and free-atom reference data," Phys. Rev. Lett. 102, 073005 (2009).

${ }^{30}$ W. Tang, E. Sanville, and G. Henkelman, "A grid-based Bader analysis algorithm without lattice bias," J. Phys.: Condens. Matter 21, 084204 (2009).

${ }^{31}$ L. S. Panchakarla, K. S. Subrahmanyam, S. K. Saha, A. Govindaraj, H. R. Krishnamurthy, U. V. Waghmare, and C. N. R. Rao, "Synthesis, structure, and properties of boron-and nitrogen-doped graphene," Adv. Mater. 21, 4726-4730 (2009). 